\title{
Preface
}

\section{Sports Medicine Statistics}

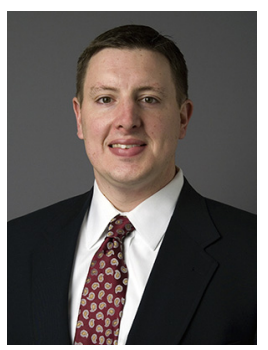

Joe M. Hart, PhD, ATC

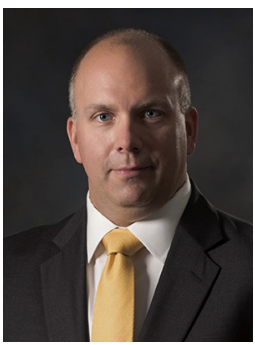

Stephen R. Thompson, MD, MEd, FRCSC Editors

As Mark Twain so famously wrote, there are lies, damn lies, and statistics. In today's age with the massive proliferation of open access, as well as predatory journals, there has never been more access to scholarly manuscripts. Consequently, it has become incumbent upon the reader to approach each study with increasing scrutiny and to question the veracity of the findings.

In addition, the research is becoming increasingly complex. A recent study was published with the subheading of "A Meta-Analysis of Randomized Controlled Trials and Systematic Review of Overlapping Systematic Reviews." A review of reviews? Dizzying!

In this present issue, we hope to present to the reader a primer on how sports medicine research and statistics are evolving into the third decade of the twenty-first century. It is our sincere hope that this provides guidance for consumers of medical research. Like any accomplished medical practitioner, successful practice relies on not only the available tools but also the manner in which they are used. Statistics are like tools in a toolbox that when used correctly can lead to discovery and innovation, however, when used incorrectly, can be misleading and potentially dangerous. In this issue, we have invited top scholars in statistics, clinical research design, mixed methodology, sports analytics, and injury prevention to provide a primer in clinical research statistics. The articles in this issue of Clinics in Sports Medicine provide an outstanding overview of contemporary approaches to research design, analysis, and data presentation that are geared toward sports medicine professionals. To lead off the issue, Dr Bryan Riemann and Dr Erin Wasserman provide outstanding fundamental reviews of biostatistics and data analytics for the sports medicine professional, after which are articles that provide statistical reviews in injury prevention, mixed model research designs, and clinical trials. Then, Dr Jay Hertel provides an excellent overview of contemporary and novel approaches to presenting data that will truly make readers consider the best approach to data visualization. These articles provide invaluable 
resources for interpretation and critical review of sports medicine research to enable decision making and evidence-based practice.

Following this, we are delighted to have one of the very rare individuals to have an eponymous outcome measure, Dr Bob Marx, and his colleagues, Dr Ruzbarsky and Dr Maron, who provide an outstanding review of the psychometric properties of patientreported outcome measures as applicable to sports medicine.

Next, Dr Wasserstein, from the University of Toronto, provides an excellent overview of how administrative databases are employed in sports medicine research. Last, one of the most influential multicenter collaborations in sports medicine has been the Multicenter Orthopedic Outcomes Network (MOON) group. Owing to their prolific output, it is important to understand how this group came about, how their research cohorts have been developed, and what lessons they have learned throughout the course of their almost 30-year history. Its founding member, Dr Kurt Spindler from the Cleveland Clinic, has provided an excellent overview of his lessons from "The MOON."

We are grateful to Dr Miller, the consulting editor, for asking us to pair up and to be able to combine the perspectives of a practicing sports medicine orthopedic surgeon alongside a full-time $\mathrm{PhD}$ researcher in orthopedic sports medicine.

Joe M. Hart, PhD, ATC

Kinesiology

Clinical Research, Orthopaedic Surgery

University of Virginia

Charlottesville, VA 22904, USA

Stephen R. Thompson, MD, MEd, FRCSC

Eastern Maine Medical Center

University of Maine

Bangor, ME 04401, USA

E-mail addresses:

joehart@virginia.edu (J.M. Hart)

theskip@gmail.com (S.R. Thompson) 